\title{
Vancomycin-Induced Drug Rash With Eosinophilia and Systemic Symptoms
}

\author{
Anne Bacal ${ }^{\mathrm{a}, \mathrm{b}}$, Tara Humphrey ${ }^{\mathrm{a}}$, Tariq Yousuf ${ }^{\mathrm{a}}$
}

\begin{abstract}
Drug rash with eosinophilia and systemic symptoms (DRESS) is a severe drug-induced hypersensitivity reaction associated with $10 \%$ mortality rate with an incidence ranging from $1 / 1,000$ to $1 / 10,000$ drug exposures. Symptoms include fever, rash, eosinophilia and multi-organ failure. DRESS has been most commonly reported with anticonvulsants, allopurinol, sulfonamides and less commonly with vancomycin. This is a case of a 34-year-old female with history of osteomyelitis being treated with vancomycin in a rehabilitation facility. After 3 weeks on vancomycin, patient started to have fever and generalized rash and was still continued on vancomycin. One week thereafter, patient was noted to be hypoxic and tachycardic, and initially admitted and treated as severe sepsis. Initial laboratory studies were remarkable for WBC $21.6 \times 10^{3} / \mu \mathrm{L}$, eosinophils $2.2 \times 10^{3} / \mu \mathrm{L}$, creatinine 1.51 $\mathrm{mg} / \mathrm{dL}$, AST 710 unit/L, ALT 659 unit/L and lactic acid $2.8 \mathrm{mg} / \mathrm{dL}$. CMV and HHV-6 were detected in serum. Chest CT showed ground glass opacities and lymphadenopathies. Liver ultrasound showed mild hepatosplenomegaly. Patient continued to be on broad spectrum antibiotics and showed no improvement. Due to worsening liver function tests and kidney injury, all antibiotics were withdrawn, after which she showed significant improvement of symptoms. During her 4 weeks of prolonged clinical course she had one relapse of symptoms with fever, rash and leukocytosis. Eventually, patient slowly recovered with supportive treatment. DRESS syndrome usually has a delayed onset with known relapse of symptoms throughout its clinical course. Addition of new drugs and herpesvirus reactivation have both been shown to cause relapse of symptoms. The mainstay of treatment remains to be withdrawal of drug and treatment with steroids. This case demonstrates DRESS syndrome secondary to vancomycin exposure with relapse of symptoms most likely associated with addition of new drugs, herpes viral reactivation and absence of steroid therapy. Vancomycin, as a commonly used drug especially with increasing MRSA, warrants increased awareness of its association with DRESS for prompt early
\end{abstract}

Manuscript accepted for publication March 19, 2015

anternal Medicine Department, University of Illinois at Chicago/Advocate Christ Medical Center, IL, USA

${ }^{b}$ Corresponding Author: Anne Bacal, Advocate Christ Medical Center, Internal Medicine Department, 4440 West 95th Street, Oak Lawn, IL 60453, USA. Email: annebacal@gmail.com

doi: http://dx.doi.org/10.14740/jmc2103w recognition of symptoms and withdrawal of offending drug.

Keywords: DRESS; Vancomycin; Drug hypersensitivity

\section{Introduction}

Drug reaction with eosinophilia and systemic symptoms (DRESS) is a rare drug-induced hypersensitivity reaction with potentially life-threatening multi-organ failure as a sequela. It has been most commonly reported with anticonvulsants and allopurinol [1], however, has also been associated with antibiotics particularly sulfonamides and less commonly with vancomycin [2]. DRESS is suspected when the presentation of symptoms occur 2 - 6 weeks after exposure to the offending drug [1]. These symptoms are usually fever, lymphadenopathies, rash and multi-organ involvement. Three scoring systems have been devised to assist clinicians in the diagnosis of DRESS for which The European Registry of Severe Cutaneous Adverse Reactions (RegiSCAR) scoring system has been most frequently used [3]. RegiSCAR devised a scoring system that identifies cases as possible, probable or definite DRESS [4]. The key underlying pathogenesis of DRESS is a drug specific immune reaction that acts as a trigger of viral reactivation by as yet unknown mechanisms; however, amplification of regulatory $\mathrm{T}$ cells has been proposed to be contributory to this viral reactivation [5]. In a study of 40 patients with DRESS, evidence of EBV, HHV-6 or HHV-7 reactivation was found in 29 patients [6]. Reactivation of herpesvirus infection coexisting with drug hypersensitivity is considered specific to DRESS [7]. In fact, the detection of HHV-6 reactivation has even been proposed as a diagnostic marker for DRESS [8]. This viral reactivation seems to further propagate $\mathrm{T}$ cell expansion particularly CD 8 cytotoxic lymphocytes [6] causing tissue damage that ends up as a vicious cycle of ongoing systemic inflammation until drug is effectively withdrawn. Hence prompt recognition of this rare syndrome and thus withdrawal of the offending drug is the mainstay of treatment. However, identification of DRESS syndrome remains to be difficult in the hospital setting as patients are often in multiple agents, onset of symptoms is delayed and there is no validated diagnostic test [9]. In this case report, we present a case of DRESS syndrome associated with vancomycin presenting like severe 


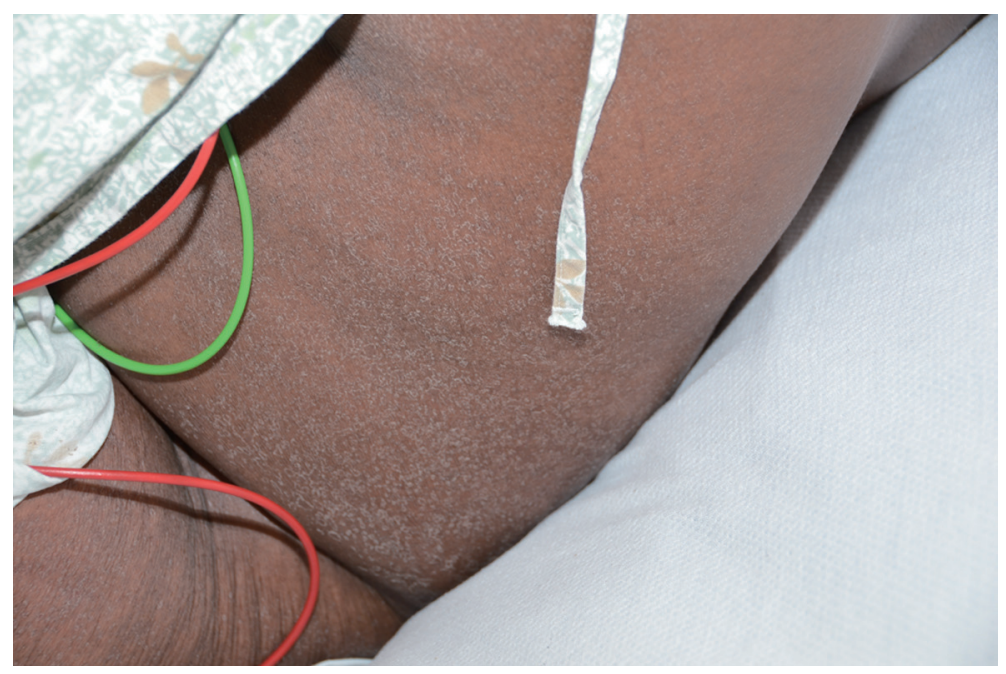

Figure 1. Hyperpigmented patches topped with diffuse desquamations in a patient with DRESS syndrome.

sepsis.

\section{Case Report}

A 34-year-old previously healthy African American female was initially admitted at our institution after suffering multiple fractures secondary to fall. She underwent several surgical repairs, and she further developed osteomyelitis. She was discharged to a rehabilitation facility where she continued to receive IV vancomycin and cefepime. After 3 weeks into vancomycin and 2 weeks on cefepime, patient started to have fever $\left(101{ }^{\circ} \mathrm{F}\right)$ and generalized erythematous papular rash. Cefepime was stopped in lieu of this rash. Her rash resolved; however, fever persisted. Four weeks into vancomycin, patient was reportedly hypoxic in the 80's, and tachycardic hence was brought back at our institution. Upon arrival, vital signs were BP 97/49, HR 108, RR 22, temp 37.7, $\mathrm{O}_{2}$ sat $97 \%$ on $2 \mathrm{~L} \mathrm{NC.}$ Physical examination revealed mild confusion, bibasal crackles, no mumurs, normal abdomen and no rash seen. She was given fluid boluses, and blood pressure was responsive. Vancomycin was shifted to daptomycin, and imipinem-cilastatin started. Laboratory studies initially revealed WBC $21.6 \times 10^{3} /$ $\mu \mathrm{L}$, eosinophils $2.2 \times 10^{3} / \mu \mathrm{L}$, platelet 264 and lymphocytes $36 \%$. Urinalysis showed protein $100 \mathrm{mg} / \mathrm{dL}$, WBC 1-5 and RBC 1-3, and no atypical lymphocytes seen on the peripheral smear. Her blood chemistry result is remarkable for $\mathrm{Cr} 1.51$ $\mathrm{mg} / \mathrm{dL}$, albumin $1.8 \mathrm{~g} / \mathrm{dL}$, AST 710 unit/L, ALT 659 unit/L, alk phos 459 unit/L and lactic acid $2.8 \mathrm{mg} / \mathrm{dL}$. Serologic test results were remarkable for CMV quantitative PCR 14,274 unit/mL and positive HHV-6 PCR, negative HIV, hepatitis A, $\mathrm{B}$ and $\mathrm{C}$, influenza, corona virus, RSV, adenovirus, enterovirus, parainfluenza virus, aspergillus galactomannan and blastomyces antigen. Blood cultures were persistently negative. Chest CT showed pulmonary edema and patchy ground glass opacities in upper and lower lobes with mediastinal, bilateral hilar and bilateral axillary lymphadenopathies with the largest lymph node being $1.4 \mathrm{~cm}$. Liver US showed mild hepatosple- nomegaly. ANA panel negative, TEE negative. Bone marrow biopsy was done and revealed eosinophilia and reactive changes. Flow cytometry showed reactive lymphocytosis. Extensive workup has basically failed to reveal a definite diagnosis. Patient continued to be febrile and tachycardic with broad spectrum antibiotics even with addition of voriconazole. Leukocytosis and eosinophilia worsened. Progressive elevation of liver function tests, worsening kidney function and onset of another rash prompted discontinuation of all antibiotics. Leukocytosis, eosinophilia, ground glass opacities on chest CT, transaminitis and acute kidney injury started to abate down slowly, and patient was no longer febrile and tachycardic. One week after this improvement, fever, tachycardia, leukocytosis but without eosinophilia recurred. Liver function tests were noted to be trending down, but total bilirubin and creatinine remained elevated. A notable desquamative type of pruritic rash was also noted (Fig. 1, 2). Consequent to this, patient had worsening liver synthetic function manifesting with jaundice, elevated INR, prolonged PTT, elevated bilirubin and low fibrinogen. Supportive therapy was maintained. At the time steroids and liver biopsy were being contemplated, patient had gradually improved on her synthetic liver function and acute kidney injury. She was at this time discharged to an acute long-term care facility in stable condition.

\section{Discussion}

DRESS syndrome is a severe drug reaction with a mortality rate up to $10 \%$ [2] with most patients dying from acute liver failure, multi-organ failure, fulminant myocarditis or hemophagocytosis [10]. Currently there are no accurate diagnostic criteria for DRESS, but it is a syndrome diagnosed by exclusion [11]. RegiSCAR scoring system has been used to grade DRESS cases as "possible", "probable" or "definite" DRESS. A delayed onset of symptoms 2 - 6 weeks after the initiation of the causative drug is a feature of DRESS more so with probable/definite cases [2]. The average time of recovery is $6-9$ weeks with 


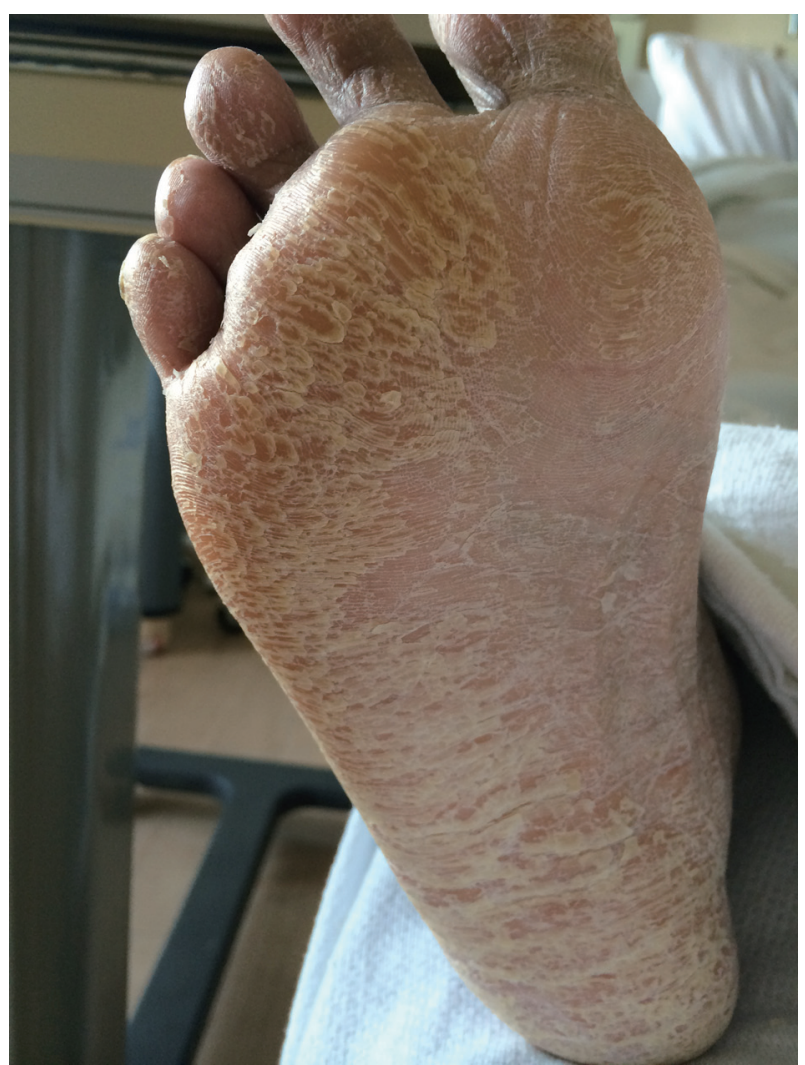

Figure 2. Multiple hyperkeratotic plaques in a patient with DRESS syndrome.

clinical course characterized by several flare-ups of symptoms [2]. It has been reported that these flares might be induced by introduction of new drugs versus simply part of the natural course of DRESS [12]. In addition, relapses have been reported associated with reactivation of HHV-6 [8] and CMV [13]. It is postulated that HHV-6 may interfere with some of the enzymes responsible for drug detoxification [14]. Our case was a "definite" DRESS achieving a RegiSCAR score of 8, and in addition other causes had been ruled out, CMV and HHV-6 reactivation were detected and the time of onset plus resolution of symptoms match the usually reported presentation of DRESS. Vancomycin was the likely causative agent considering timing of onset of symptoms after initiation of drug. This case also describes flare-ups during the clinical course. It is unknown if prompt initiation of steroids in our patient might have caused a more rapid recovery for DRESS syndrome as one study have shown a notable symptomatic and laboratory improvement after 3 days of completing $48 \mathrm{~h}$ of steroid treatment [3]. Although DRESS has been less commonly reported due to vancomycin compared to more common drugs such as anticonvulsants and allopurinol, DRESS cases related to vancomycin are rising in numbers likely from increased use for MRSA and continuous IV infusions leading to higher trough levels and hence toxicity of vancomycin [9]. Prompt diagnosis and treatment, most importantly identifying and stopping the drug, remain to be the most important step in the management of DRESS syndrome.

\section{Conclusion}

We report a case of DRESS syndrome associated with a high risk medication, vancomycin with clinical course complicated with flare of symptoms even after stopping drug. Drug exposure also overlaps with several other drugs like cefepime, daptomycin, imipenem-cilastatin, moxifloxacin and voriconzole; however, the timeline that has been described for DRESS most especially fits vancomycin exposure in this patient. This case also demonstrated reactivation of herpesvirus that has been reportedly associated with DRESS syndrome. It is possible that the addition of other drugs together with herpesvirus reactivation and absence of steroid therapy all contributed to this patient's prolonged and complicated clinical course and associated relapse. Vancomycin, as a commonly used drug especially with increasing MRSA, warrants increased awareness of its association with DRESS, since it can prompt early recognition of symptoms and withdrawal of offending drug.

\section{Conflict of Interest}

No conflict of interest to report.

\section{References}

1. Kardaun SH, Sekula P, Valeyrie-Allanore L, Liss Y, Chu $\mathrm{CY}$, Creamer D, SidoroffA, et al. Drug reaction with eosinophilia and systemic symptoms (DRESS): an original multisystem adverse drug reaction. Results from the prospective RegiSCAR study. Br J Dermatol. 2013;169(5):1071-1080.

2. Cacoub P, Musette P, Descamps V, Meyer O, Speirs C, Finzi L, Roujeau JC. The DRESS syndrome: a literature review. Am J Med. 2011;124(7):588-597.

3. Guleria VS, Dhillon M, Gill S, Naithani N. Ceftriaxone induced drug rash with eosinophilia and systemic symptoms. J Res Pharm Pract. 2014;3(2):72-74.

4. Kardaun SH, Sidoroff A, Valeyrie-Allanore L, Halevy S, Davidovici BB, Mockenhaupt M, Roujeau JC. Variability in the clinical pattern of cutaneous side-effects of drugs with systemic symptoms: does a DRESS syndrome really exist? Br J Dermatol. 2007;156(3):609-611.

5. Takahashi R, Kano Y, Yamazaki Y, Kimishima M, Mizukawa Y, Shiohara T. Defective regulatory T cells in patients with severe drug eruptions: timing of the dysfunction is associated with the pathological phenotype and outcome. J Immunol. 2009;182(12):8071-8079.

6. Picard D, Janela B, Descamps V, D'Incan M, Courville P, Jacquot S, Rogez S, et al. Drug reaction with eosinophilia and systemic symptoms (DRESS): a multiorgan antiviral T cell response. Sci Transl Med. 2010;2(46):46ra62.

7. Descamps V, Valance A, Edlinger C, Fillet AM, Gros$\sin$ M, Lebrun-Vignes B, Belaich S, et al. Association of human herpesvirus 6 infection with drug reaction with eosinophilia and systemic symptoms. Arch Dermatol. 2001;137(3):301-304.

8. Tohyama M, Hashimoto K, Yasukawa M, Kimura H, 
Horikawa T, Nakajima K, Urano Y, et al. Association of human herpesvirus 6 reactivation with the flaring and severity of drug-induced hypersensitivity syndrome. Br J Dermatol. 2007;157(5):934-940.

9. Young S, Ojaimi S, Dunckley H, Douglas MW, Kok J, Fulcher DA, Lin MW, et al. Vancomycin-associated drug reaction with eosinophilia and systemic symptoms syndrome. Intern Med J. 2014;44(7):694-696.

10. Eshki M, Allanore L, Musette P, Milpied B, Grange A, Guillaume JC, Chosidow O, et al. Twelve-year analysis of severe cases of drug reaction with eosinophilia and systemic symptoms: a cause of unpredictable multiorgan failure. Arch Dermatol. 2009;145(1):67-72.

11. Sriratanaviriyakul N, Nguyen LP, Henderson MC, Al- bertson TE. Drug reaction with eosinophilia and systemic symptoms syndrome (DRESS) syndrome associated with azithromycin presenting like septic shock: a case report. J Med Case Rep. 2014;8:332.

12. Tetart F, Picard D, Janela B, Joly P, Musette P. Prolonged evolution of drug reaction with eosinophilia and systemic symptoms: clinical, virologic, and biological features. JAMA Dermatol. 2014;150(2):206-207.

13. Mathuram A, George R. Cytomegalovirus Reactivation in drug induced hypersensitivity syndrome. J Assoc Physicians India. 2014; 62:533-535.

14. Ichiche M, Kiesch N, De Bels D. DRESS syndrome associated with HHV-6 reactivation. Eur J Intern Med. 2003;14(8):498-500. 